\title{
Comparação de diluidores comerciais na motilidade, funcionalidade e integridade da membrana plasmática do espermatozoide bovino*
}

\section{Comparison of commercial diluents on motility, functionality and integrity of plasma membrane of bovine spermatozoa}

\author{
Monalyse Kevelyn Borges de Oliveira, ${ }^{* *}$ João Pedro Brandão Zandonaide, ${ }^{* \star *}$ Neimar Correa Severo, ${ }^{* \star * *}$ \\ Antonieta Lourenia Gomes, ${ }^{* \star *}$ Mauricio Scoton Igarasi, ${ }^{* \star * \star * *}$ Amanda Pifano Neto Quintal, ${ }^{* * \star * \star}$ André Belico Vasconcelos ${ }^{\star \star \star * \star *}$
}

\begin{abstract}
Resumo
O objetivo do estudo foi comparar o efeito de três diluidores comerciais (Tryladi ${ }^{\circledR}$, Botu-Bov ${ }^{\circledR}$ e OptiXcell ${ }^{\circledR}$ ) na qualidade do espermatozoide bovino após o processo de criogenia. Para tal, foram utilizados oito touros da raça Nelore (2 ejaculados/touro). As amostras de sêmen fresco, diluído e pós-descongelamento foram avaliadas, comparando os parâmetros de motilidade total, vigor, funcionalidade da membrana (HOST) e integridade da membrana (eosina). Os dados foram expressos em média e desvio padrão. As variáveis foram submetidas às análises de ANOVA e Tukey ou teste de Friedman e Dunn's, dependendo da normalidade $(p<0,05)$. Os achados mostram que no momento da diluição não houve diferença $(p>0,05)$ entre os diluidores comerciais nos parâmetros avaliados (exceto integridade da membrana plasmática). No entanto, no momento do pós-descongelamento os espermatozoides criopreservados utilizando-se o diluidor Tryladil ${ }^{\circledR}$ apresentaram maiores valores $(p<0,05)$ referentes a integridade $e$ funcionalidade da membrana plasmática comparado aos diluídos em Botu-Bov ${ }^{\circledR}$ e OptIXcell ${ }^{\circledR}$. Os parâmetros relacionados a cinética espermática (motilidade e vigor) não se diferiram $(p>0,05)$ entre os diluidores comerciais utilizados. Em conclusão, no momento pós-descongelamento o diluidor Tryladil ${ }^{\circledR}$ apresentou os melhores resultados nos parâmetros de integridade e funcionalidade da membrana plasmática. Sendo assim, recomenda-se o diluidor Tryladil ${ }^{\circledR}$ para criopreservação de sêmen de bovinos da raça Nelore.
\end{abstract}

Palavras-chave: criopreservação, teste HOST, diluente, touro, Nelore.

\begin{abstract}
The main of the study was to compare the quality of frozen bull semen processed with three different commercially extenders

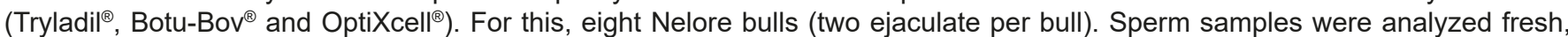
diluted and frozen-thawed. The parameters analyzed were total motility, sperm vigor, functional integrity of sperm plasma membrane (HOST) and plasma membrane integrity (eosin). Date were expressed as mean and standard deviation. The variables were subjected to ANOVA (Tukey test) or Friedman (Dunn's test) test according to normality $(p<0,05)$. The results indicate that there was no difference $(p>0,05)$ among all treatments in the parameters evaluated (except plasma membrane integrity) at dilution moment. However, Tryladil extender promoted an increase $(p<0,05)$ in functional and integrity of sperm plasma membrane compared with others extenders at the post-thawing analyze. After thawing, there was no difference $(p>0,05)$ among all treatments in the kinetic parameters. In conclusion, the Tryladil ${ }^{\circledR}$ extender promoted an increase in functional and integrity of frozen-thawed sperm plasma membrane. Therefore, the Tryladil ${ }^{\circledR}$ extender is recommended to be use as an extender for Nelore bull sperm cryopreservation.
\end{abstract}

Keywords: cryopreservation; HOST test, diluent, bull, Nelore.

\section{Introdução}

A utilização do sêmen bovino criopreservado apresenta vantagens em relação às demais formas de processamento pois possibilita a expansão da genética de diferentes touros mundialmente, além de atuar na prevenção da disseminação de doenças passíveis de transmissão por meio de monta natural. No entanto, o uso do sêmen criopreservado geralmente apresenta menores taxas de fertilidade em relação ao sêmen fresco (Bertol et al., 2014).

Os principais fatores envolvidos nessa queda de fertilidade são as mudanças de temperatura durante o processamento, o estresse osmótico e tóxico representado pela exposição aos crioprotetores e a formação e dissolução de cristais de

*Recebido em 5 de julho de 2018 e aceito em 25 de novembro de 2018.

**Discente do Curso de Medicina Veterinária da Universidade de Uberaba, UNIUBE.

***Discente do Programa de Pós Graduação em Sanidade e Produção Animal nos Trópicos, UNIUBE.

****Central de Produção de Sêmen, Alta Genetics.

*****Docente da Faculdade Associada de Uberaba, FAZU.

******Docente do Programa de Pós-Graduação em Sanidade e Produção Animal nos Trópicos, UNIUBE. 
gelo extracelulares (Oliveira et al., 2013; Bertol et al., 2014; Quan et al., 2016; Losano, 2016). Assim a sobrevivência do sêmen criopreservado, depende de agentes que presentes na composição do meio diluidor, crioprotetores, tornando-se um fator crucial para a preservação da integridade celular e sucesso da fertilização após o congelamento (Leite et al, 2011).

Os componentes essenciais dos diluidores para criopreservação são: as (1) substâncias iônicas e não iônicas (responsáveis pela manutenção da osmolaridade e tamponamento); (2) as fontes de lipoproteínas de alto peso molecular (necessárias para a prevenção do choque frio, como gema de ovo e leite); (3) os crioprotetores intracelulares (glicerol, etilenoglicol e DMSO); (4) as fontes energéticas (representadas por açúcares, como glicose e frutose); e os demais aditivos, tais como antibióticos, enzimas, antioxidantes e detergentes (Foote et al.,2002; Aguiar et al., 2007; Bertol et al., 2014; Vasconcelos et al., 2017).

Dentre os diluidores comercias utilizados para a criopreservação de sêmen bovino destacam-se três: Tryladil ${ }^{\circledR}$, OptiXcell ${ }^{\circledR}$ e Botubov $^{\circledR}$. O Tryladil ${ }^{\circledR}$ é um diluidor concentrado para preparação de meio para congelamento de sêmen contendo açúcar, TRIS, ácido cítrico, tampões, antibióticos, gema de ovo, glicerol e água pura. OptiXcell ${ }^{\circledR}$ composto por carboidratos, sais, antibióticos, antioxidantes, glicerol e fosfolipídios organizados em lipossomos, sendo vesículas de fosfolipídios preparadas artificialmente e solúveis em água. O Botubov ${ }^{\circledR}$ possui em sua composição: açucares, antioxidantes, aminoácidos, gema de ovo e glicerol (Informação do fabricante).

Para minimizar os efeitos deletérios da criopreservação, inúmeros diluentes e crioprotetores vem sendo testados, além de aditivos para proteger as células e fornecer substratos para sua manutenção durante e após a congelação (Leite et a., 2011). Desta forma, o objetivo desde estudo foi comparar o efeito de três diluidores comerciais Tryladil ${ }^{\circledR}$, Botu-Bov ${ }^{\circledR}$ e OptiXcell ${ }^{\circledR}$ na motilidade total, vigor espermático, integridade e funcionalidade da membrana plasmática do espermatozoide bovino Nelore antes (momento da diluição) e após o descongelamento.

\section{Material e métodos}

O Projeto foi aprovado no Comitê de Ética em Experimentação Animal da Universidade de Uberaba (CEEA-045/2017) e realizado na central de coleta e processamento de sêmen bovino Alta Genetics Brasil, localizada no município de Uberaba - Minas Gerais, durante agosto a setembro de 2017.

Foram utilizados oito touros da raça Nelore apresentando idade entre 6 -10 anos, peso corpóreo médio em torno de $1.100 \mathrm{~kg}$ e escore corporal entre 3-4. Os parâmetros mínimos das amostras de sêmen para o presente estudo foram: motilidade total $(\leq 60 \%)$, vigor espermático $(\leq 3)$, e concentração espermática $\left(\leq 6 \times 10^{8} /\right.$ $\mathrm{ml}$ ). A alimentação dos animais foi constituída por silagem de milho e bagaço de cana de açúcar. Um total de 16 ejaculados (dois por touro) foram coletados pelo método de vagina artificial (modelo - IMV Technologies ${ }^{\circledR}$ ). Após a coleta, os ejaculados foram imediatamente encaminhados ao laboratório para serem analisados e posteriormente submetidos ao processamento para criopreservação. Para controle interno da reação, as amostras de sêmen in natura foram avaliadas para motilidade total, vigor espermático, integridade e funcionalidade da membrana espermática.
Objetivando alcançar a concentração espermática de $3 \mathrm{x}$ $10^{7}$ de SPTZ/dose, o sêmen foi diluído utilizando três diluentes

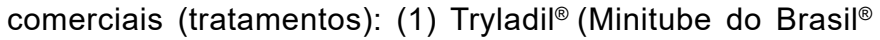
Ltda.); (2) Botu-Bov ${ }^{\circledR}$ (Botupharma ${ }^{\circledR}$ Biotecnologia Animal) e (3) OptiXcell ${ }^{\circledR}\left(\right.$ IMV $^{\circledR}$ Technologies). As análises foram realizadas em dois momentos: (1) Diluição e (2) Pós-descongelamento.

As amostras de sêmen diluídas de acordo com seu respectivo grupo experimental foram colocadas em um tubo de polipropileno $(50 \mathrm{~mL})$ e estes foram depositados em frascos contendo água a $37^{\circ} \mathrm{C}$, visando evitar o choque térmico. Estas amostras foram encaminhadas para câmara fria $\left(4^{\circ} \mathrm{C}\right)$, onde permaneceram por três horas para resfriamento.

Após a etapa de resfriamento, as amostras foram então envasadas em palhetas de $0,25 \mathrm{~mL}$ (IMV $\left.{ }^{\circledR} T e c h n o l o g i e s\right)$ identificadas com o nome do touro, partida do ejaculado e diluidor utilizado. Para a curva de estabilização em vapor de nitrogênio líquido, utilizou-se uma caixa isotérmica convencional de 50L. As palhetas foram dispostas em grade de metal, e mantidas a três centímetros acima do nível do nitrogênio, durante 20 minutos. Posteriormente, as palhetas foram imersas no nitrogênio líquido $\left(-196^{\circ} \mathrm{C}\right)$ e transferidas para botijões criogênicos, e mantidas até o momento da análise.

As amostras foram descongeladas em banho-maria a $37^{\circ} \mathrm{C}$ por 30 segundos e homogeneizadas em tubos de ensaio aquecidos a $37^{\circ} \mathrm{C}$ para posterior avaliação. $\mathrm{O}$ ejaculado foi avaliado macroscopicamente e microscopicamente após a diluição do sêmen fresco e também após o processo de criogenia de todos os grupos experimentais.

Para avaliação macroscópica foram avaliados os seguintes parâmetros: volume (estimado por balança de precisão, no qual pesa-se um tubo tipo Falcon $15 \mathrm{~mL}$, tara a balança e posteriormente pesa-se o tubo mais ejaculado, cujo valor é registrado em gramas e transformado em mililitros), cor e odor. $\mathrm{Na}$ avaliação microscópica, os seguintes parâmetros foram avaliados: motilidade total, vigor espermático, concentração espermática, funcionalidade da membrana espermática e integridade da membrana espermática. A concentração espermática foi estimada por espectrofotometria (Genesys $20^{\circledR}$ ), utilizando o comprimento de onda 550nm. Para tal foi pipetado $50 \mu \mathrm{L}$ da amostra do ejaculado em cubeta e esta preenchida com sete $\mathrm{mL}$ de solução Formol-Salina (solução fisiológica 0,9\% e formol na concentração de 1\%).

A motilidade total e vigor espermático (força de locomoção dos espermatozoides; escore de 0 a 5) foram avaliados inserindo 10 $\mu \mathrm{L}$ de sêmen em uma lâmina previamente aquecida $\left(37^{\circ} \mathrm{C}\right)$, em mesa aquecedora portátil, modelo MTP2030 (NeoVet) e coberta por uma lamínula. A análise foi realizada em microscopia de campo claro em aumento de 10x, conforme manual do colégio Brasileiro de reprodução (CBRA, 2013). A funcionalidade da membrana espermática foi avaliada pelo teste o hiposmótico (HOST). Para isso, $20 \mu \mathrm{L}$ de sêmen foram incubados a $37^{\circ} \mathrm{C}$ por 60 minutos em uma solução $150 \mathrm{mOsm} / \mathrm{Kg}$ contendo D-frutose $(13,5 \mathrm{~g} / \mathrm{L}$; Sigma) e citrato de sódio (7,3g/L; Sigma)(Correa e Zavos, 1994). Após o período de incubação, as amostras foram fixadas em $0,5 \mathrm{~mL}$ de formol salino. Em seguida, $10 \mu \mathrm{L}$ da solução foi colocada em uma lâmina e coberta por lamínula. Posteriormente, 100 espermatozoides foram classificados de acordo com presença ou ausência do dobramento/enrolamento da cauda em microscopia óptica (aumento de 1000x). O cálculo 
da resposta osmótica foi determinado pela porcentagem de células reativas ao teste hiposmótico $(\% \mathrm{HOST})=(\%$ de alterações na região de cauda após teste HOST) - (\% de alterações na região de cauda dos espermatozoides antes do teste HOST) (Melo e Henry, 2005; Vasconcelos et al., 2017).

A integridade de membrana plasmática foi avaliada pelo teste de coloração com eosina (Sigma - Aldrich Corp.). Para isso, $10 \mu \mathrm{L}$ de sêmen e $10 \mu \mathrm{L}$ de eosina (1\%p/v) previamente aquecidos (37 ${ }^{\circ} \mathrm{C}$ ) foram depositados em lâmina para realização de esfregaço. A lâmina foi mantida a $37^{\circ} \mathrm{C}$ durante 1 minuto objetivando a sua secagem. Seguidamente, procedeu-se a contagem de 100 células em microscopia óptica (40x) durante cinco minutos após a elaboração do esfregaço (Blom, 1950; Vasconcelos et al., 2017).

Para análise estatística foi utilizado o programa GraphPadPrism 6.0 (GraphPad Software Inc., San Diego, CA, USA), com nível de significância foi de $5 \%$.Valores de média e desvio padrão das características espermáticas (motilidade, vigor, host e eosina) foram calculadas, logo após a diluição do sêmen in natura e posteriormente ao descongelamento do sêmen, dos três diluidores comerciais utilizados. A mesma amostra de sêmen foi pareada na avaliação antes e após o processo de criogenia. Para todos os grupos, inicialmente foi realizado o teste de normalidade Shapiro-Wilk e teste para verificar a homogeneidade das variâncias (teste de Cochran). Dados com distribuição normal, como HOST, foram analisados pelo teste estatístico ANOVA seguido pelo teste Tukey. Dados não paramétricos com variâncias homogêneas de motilidade, vigor e eosina foram analisados pelo teste Friedman seguido pelo teste Dunn's.

\section{Resultados e discussão}

A avaliação do sêmen in natura apresentou os seguintes resultados: motilidade total $(71,6 \%)$, vigor espermático $(4,8)$, funcionalidade $(77 \%)$ e integridade da membrana $(84,4 \%)$ espermática. Os valores referente a motilidade total e vigor espermático encontram-se dentro do padrão recomendado pelo CBRA (2013). Martins et al (2011) avaliaram ejaculados de touros da raça Nelore $(n=6)$ e observaram valores próximos ao presente estudo: $60,3 \%$ (funcionalidade da membrana plasmática; HOST) e $77,2 \%$ (integridade da membrana plasmática; teste de coloração com eosina).

Os resultados referentes aos parâmetros analisados neste estudo encontramse na Tabela 1. No momento da diluição, não houve diferença $(p>0,05)$ entre os tratamentos nos parâmetros avalia-dos (motilidade total, vigor, integridade e funcionalidade da membrana plasmática).

Os resultados encontrados em todos os diluidores para o aspecto motilidade no momento do pós-descongelamento Descongelados). corroboram com o descrito na literatura (Leite et al., 2011; Janett et al., 2005). Na avaliação do sêmen após o descongelamento não houve diferença $(p>0,05)$ entre os diluidores comerciais nos parâmetros de motilidade total e vigor espermático. No entanto, os espermatozoides diluídos no Tryladil $^{\circledR}$ apresentaram maior $(p<0,05)$ taxa de integridade e funcionalidade da membrana plasmática quando comparado aos diluídos no Botubov ${ }^{\circledR} \mathrm{e}$ Optixcell ${ }^{\circledR}$.

O possível efeito do Tryladil $^{\circledR}$ sobre a membrana plasmática pode ocorrer devido a associação do glicerol com componentes do diluidor, como a gema de ovo, cujo principal benefício para a membrana plasmática é fornecer uma fração LDL que previne a perda de fosfolipídios da membrana, aumentando assim a tolerância ao choque térmico e ao processo criopreservação. Entretanto a razão molar entre os constituintes e a concentração celular pode ser um fator preponderante sobre a função desta molécula (Layek, et al. 2016).

Para avaliar o efeito do processo de criopreservação na qualidade espermática foram realizadas comparações entre o momento da diluição e pós-descongelamento em cada diluidor comercial utilizado neste estudo. Considerando o diluidor Tryladil ${ }^{\circledR}$, o sêmen após descongelamento apresentou uma redução $(p<0,05)$ nos parâmetros analisados em comparação ao sêmen diluído. Em relação aos diluidores Botubov ${ }^{\circledR}$ e Optixcell ${ }^{\circledR}$ exceto o parâmetro de vigor espermático não apresentou redução $(p>0,05)$ entre o sêmen diluído e sêmen após o descongelamento.

Ao analisar isoladamente as avaliações para cada diluidor antes a após o descongelamento, pode-se observar que a motilidade total e vigor, sofreram variações durante o processo de congelamento do sêmen, com uma queda de aproximadamente de $35 \%$ para todos os diluidores. Watson (1995) relata que a diminuição da qualidade espermática, póscriopreservação poderia ficar entre $40 \%$ a $50 \%$ do ejaculado. A literatura registra que o objetivo da criopreservação do sêmen é manter a viabilidade espermática por meio da redução do metabolismo e preservação dos componentes (Peeg, 2002; Silva e Guerra, 2011). Entretanto, as variações de temperatura dentro do contexto bioquímico promovem variações fisiológicas na membrana, diminuindo a viabilidade, além de atuar em fatores físico-químicos relacionados ao $\mathrm{pH}$ e tensão de salinidade (Neidleman, 1987). Para Layek et al (2016) o processo de

Tabela 1: Média e desvio padrão dos parâmetros motilidade (\%), vigor (Score de 1 a 5), funcionalidade da membrana (HOST) e integridade da membrana (eosina) nos diluidores comerciais avaliados antes do congelamento (Diluídos) e após o processo de criogenia (Descongelado) do sêmen bovino Nelore

\begin{tabular}{lcccccc}
\hline \multirow{2}{*}{ Parâmetros } & \multicolumn{2}{c}{ TRYLADIL $^{\circledR}$} & \multicolumn{2}{c}{ BOTUBOV $^{\circledR}$} & \multicolumn{2}{c}{ OPTIXCELL $^{\circledR}$} \\
& Diluídos & Descongelado & Diluídos & Descongelado & Diluídos & Descongelado \\
\hline Motilidade (\%) & $71,2 \pm 6,9^{\mathrm{aA}}$ & $48,7 \pm 7,7^{\mathrm{bA}}$ & $71,2 \pm 6,9^{\mathrm{aA}}$ & $51 \pm 8,5^{\mathrm{bA}}$ & $70,3 \pm 6,9^{\mathrm{aA}}$ & $49,3 \pm 8,6^{\mathrm{bA}}$ \\
Vigor (1 a 5) & $4,8 \pm 0,4^{\mathrm{aA}}$ & $4 \pm 0,5^{\mathrm{bA}}$ & $4,8 \pm 0,4^{\mathrm{aA}}$ & $4,3 \pm 0,6^{\mathrm{aA}}$ & $4,8 \pm 0,4^{\mathrm{aA}}$ & $4,1 \pm 0,6^{\mathrm{aA}}$ \\
HOST (\%) & $64,9 \pm 9,3^{\mathrm{aA}}$ & $49,1 \pm 7,7^{\mathrm{bA}}$ & $62,5 \pm 8,4^{\mathrm{aA}}$ & $40,6 \pm 7,8^{\mathrm{bB}}$ & $67,2 \pm 12,1^{\mathrm{aA}}$ & $44,3 \pm 9,1^{\mathrm{bAB}}$ \\
Eosina (\%) & $83,2 \pm 6,6^{\mathrm{aA}}$ & $67,5 \pm 8,4^{\mathrm{bA}}$ & $83 \pm 5,5^{\mathrm{aA}}$ & $56,4 \pm 9,1^{\mathrm{bB}}$ & $79 \pm 6,9^{\mathrm{aB}}$ & $54,1 \pm 5,8^{\mathrm{bB}}$ \\
\hline
\end{tabular}

Letras minúsculas indicam a avaliação das médias entre os mesmos diluidores (Diluídos vs Descongelado). Letras maiúsculas indicam avaliação das médias entre os diluidores diferentes (Diluídos vs Diluídos) e (Descongelados vs 
criogenia causa a translocação de lipídeos e a desestabilização de membrana o que pode levar a perda da integridade da membrana e também uma reação acrossomal prematura. Crespilho (2006) e Chaudhari et al (2015) estabelecem que com a queda da temperatura os lipídeos passam do estado fluido para o estado gel onde as cadeias de ácidos graxos se desordenam, até essas estruturas emparelham-se e se tornam rígidas e fracas sujeitas a danos.

Leite e colaboradores (2011) apontam que na criopreservação do sêmen bovino, os diluidores à base de gema de ovo são os mais comuns por apresentarem melhores resultados. Contudo ao analisar os diluidores a base de gema de ovo Tryladil $^{\circledR} \mathrm{e}$ Botu-Bov $^{\circledR}$, estes apresentaram resultados distintos em relação a funcionalidade e integridade da membrana espermática. Os aspectos físico-químicos de cada diluidor, apesar de apresentarem em sua composição substancias similares, a razão concentração/ célula é diferente. Desta forma a concentração molar de cada um dos constituintes presentes no diluidor Tryladil ${ }^{\circledR}$ se ajustaram melhor ao protocolo usado na central de produção de sêmen, quanto a curva de congelamento, tempo de equilíbrio, justificando melhor qualidade de membrana, após o processo de criogenia.

Portanto, conclui-se que o Tryladil $^{\circledR}$ é o diluidor indicado a ser utilizado para criopreservação de sêmen de bovinos da raça Nelore visto que este apresentou melhores resultados para os parâmetros de integridade e funcionalidade da membrana plasmática no momento do pós-descongelamento.

\section{Agradecimentos}

À empresa Alta Genetics Brasil por nos conceder seus animais para o desenvolvimento deste estudo. À Fundação de Amparo da Pesquisa do Estado de Minas Gerais (FAPEMIG) e a Coordenação de Aperfeiçoamento de Pessoal de Nível Superior - Brasil (CAPES) - Código de Financiamento 001.

\section{Referências}

AGUIAR, P. R. L.; MORAES, J.B.; MALSCHITZKY. E.; SILVA, A.C. Criopreservação de sêmen bovino utilizando diluente à base de PBS com três diferentes percentuais de gema de ovo. Veterinária em Foco, v. 5, n. 1, p. 44-50, 2007.

ANSARI, M.S.; RAKHA, B.A.; AKHTER, S. Cryopreservation of bull semen in OptiXcell and convencional extenders: Comparison of semen quality and fertility. Animal Science PapersandReports. v. 35, n. 3, p. 317-328, 2017.

ARRUDA, R.L.; ORRO, I.R.; PASSOS, T.S.; COSTA E SILVA, E.V.; ZÚCCARI, C.E.S.N. Técnicas para avaliaçãolaboratorial da integridadeestrutural e funcional do sêmen congelado de touros. Revista Brasileira de Reprodução Animal, v.34, p.168-184, 2010.

BLOM, E. A one-minute live-dead sperm stain by means of eosinnigrosin. Fertily and Sterility, v. 1, n. 2, p. 176-177, 1950.

BERTOL, M. A. F.; WEISS, R. R.; FUJITA, A. S.; KOZICHI, L. E.; ABREU, A. C. M. R.; PEREIRA, J. F. S. Dois diluentes comerciais na criopreservação de espermatozoides do epidídimo de touros. Ciência Rural, v.44, n. 9, p. 1658-1663, 2014.

CBRA. Manual para Exame Andrológico e Avaliação de Sêmen Animal. 3a ed. Colégio Brasileiro de Reprodução Animal, Belo Horizonte. 104p, 2013.

CHAUDHARI, D.V.; DHAMI,A.J.; HADIYA, K.K.; PATE, J.A. Relative efficacy of egg yolk and soya milk-based extenders for cryopreservation $\left(-196^{\circ} \mathrm{C}\right)$ of buffalo semen, Veterinary Worl, v. 8(2), p.239-244, 2015.

CRESPILHO, A.M.; PAPA, F.O.; KALBERTI,K.; SIQUEIRA FILHO, E.R.; MARTINS Jr. A.; NOVAES, J. L. C.; DELL'AQUA, J.A.Eficiência comparativa entre dois diluidores para a congelação de sêmen bovino sobre os padrões de motilidade e integridade de membrana plasmática. ArsVeterinaria, Jaboticabal, $S P$, v. 22, n.3, p.229-235, 2006.

FOOTE, R. H.; BROCKETT, C. C.; KAPROTH, M. T. Motility and fertility of bull sperm in whole milk extender containing antioxidants. Animal Reproduction Science, v.71, p.13-23, 2002.

INSKEEP, P.B. \& HAMMERSTEDT, R.H. A colorimetric method to assess endogenous metabolism and its application to the study of bovine sperm. Journal of Biochemical and Biophysical methods, v.7, p. 199-210, 1983
JANETT F., KEO S., BOLLWEIN H., HÄSSIG M., THUN R. Comparison of AndroMed $\AA$, Bioxcell and Triladyl $®$ extender for cryopreservation of bull semen. Conference on Physiology and Pathology of Reproduction; Zürich, February 2005.

JASKO, D.J. Procedures for cooling and freezing of equine semen. Ars Veterinaria, v.10, n.2, p.152-165, 1994.

LAYEK, S. S.; MOHANTY T. K.; KUMARESAN, A.; PARKS J. E.Cryopreservation of bull semen: Evolution from egg yolk based to soybean based extenders.Animal Reproduction Science, v.172, p.1-9, 2016.

LEITE, P. A.; SCHREDER, G. G.; ALMEIDA, C. L. R. A.; ZUCCARI, C. E. S. N.; COSTA E SILVA, E. V. Criopreservação do sêmen bovino.Cient. Ciênc. Biol. Saúde. v. 13, n. 4, p. 279-286, 2011.

LOSANO, J. D. A. Utilização de pentoxifilina e antioxidantes na criopreservação do sêmen bovino: qualidade seminal e estresse oxidativo.ArsVeterinaria, Jaboticabal, SP, v.32, n.2, p.110-116, 2016.

MARTINS, L.F.; PINHO, R.O.; PARAIZO, R.M.; OLIVEIRA, R.R.; CASTILHO, E.F.; GUIMARÃES, J.D. Avaliação de diferentes osmolaridades de soluções hiposmóticas e tempos de incubação no teste hiposmótico do sêmen de touros Nelore. Revista Brasileira de Zootecnia, v. 40, n. 7, p. 1519-1525, 2011.

MEDEIROS, C.M.O.; FORELL, F.; OLIVEIRA, A.T.D.; RODRIGUEZ, J.L. Current status of sperm cryopreservation: why isn't better? Theriogenology 57:327-34, 2002.

MELO, M. I. V.; HENRY, M. Teste hiposmótico na avaliação da viabilidade do sêmen equino resfriado com diferentes diluidores. Arquivo Brasileiro de Medicina Veterinária e Zootecnia, v. 57, n. 6, p. $757-763,2005$.

NEIDLEMAN, S.L Effects of Temperature on Lipid Unsaturation, Biotechnology and Genetic Engineering Reviews, 5:1, 245-268, 1987 DOI: $10.1080 / 02648725.1987 .10647839$

OLIVEIRA, G.C.;OLIVEIRA, B.M.M.; CELEGHINI, E.C.C.; FERNANDES, C.B.; MATTOS, C.B.;Criopreservação do sêmen equino: uma revisão. Rev. Bras. Reprod. Anim, v.37, n.1, p.23-28, 2013.

PEGG, D.E. The History and Principles of Cryopreservation. Seminars in Reproductive Medicine, v.20, n.1, p.05- 14, 2002. 
PRADO, R. B.; KOIVISTO, M. B.; CARREIRA, J. T.; PERRI, S. H. V.; RODRIGUES, L. H.; ANTIQUE NETTO, H.; TORREGROSSA, T. L. G.; VICENTE, W. R. R.; FELICIANO, M. A. R. Efeito da utilização de diferentes diluidores para produção in vitro de embriões bovinos. Arquivo Brasileiro de Medicina Veterinária e Zootecnia, v. 64, n. 5, p. 1118-1126, 2012.

QUAN, B. G.; WU, G. Q.; WANG, Y. J.; LI, D. J.; QIONG, H. $H$. Effects of the Tris, Tes, or skim milk based extender on in vitro parameters of ram spermatozoa during liquid storage. SmallRuminantResearch,v. 134, p.14-21, 2016.

SHIUA, W.Y.; MAB, K.C. Temperature Dependence of Physical-Chemical Properties of Selected Chemicals of Environmental Interest. I. Mononuclear and Polynuclear Aromatic Hydrocarbons.J. Phys. Chem. Ref. Data, v. 29, n. 1, 2000.

SILVA, S.V.; GUERRA, M.M.P. Efeitos da criopreservação sobre as células espermáticas e alternativas para redução das crioinjúrias. Rev. Bras. Reprod. Anim., Belo Horizonte, v.35, n.4, p.370-384, 2011.
VASCONCELOS, A.B.; SOUZA, P.C.; VARAGO, F.C.; LAGARES, M.A.; SANTORO, M.M. Determination of Optimal Glucose Concentration for Microcalorimetric Metabolic Evaluation of Equine Spermatozoa. Brazilian Archives of Biology and Technology Vol.52, n. 5: p.1129-1136, 2009.

VASCONCELOS, A. B.;ZANDONAIDE, J. B. Z.;SOBRINHO, A. L. F.;SILVA, B. O.;QUINTAL, P. N. Q. A comparative study of three different dyes evaluating the physical integrity of the plasma membrane of cryopreserved bovine spermatozoa. Vet. Not. v.23, p.13-2017.

WATSON, P.F. Recent developments and concepts in the cryopreservation of spermatozoa and the assessment of their postthawing function. ReproductionFertilityandDevelopment, v.7, p. 871-891, 1995. 